\title{
Siguiendo los rastros de los depósitos rituales: esbozo de un marco arqueológico para el estudio de las prácticas rituales en Palenque
}

\author{
Tracing the Ritual Deposits: \\ Establishing an Archaeological Framework \\ for the Study of Ritual Practices in Palenque
}

\author{
Lisa M. JoHnSON \\ University of California, Berkeley
}

\begin{abstract}
Resumen: Diversas excavaciones arqueológicas desarrolladas en las Tierras Bajas del sureste de México, Guatemala y Belice han revelado patrones generalizados en las prácticas rituales, incluso en el tipo de materiales utilizados en ofrendas rituales y entierros humanos colocados dentro de los pisos y plazas de templos públicos y casas privadas. Sin embargo, las evidencias materiales de eventos rituales pasados parecen ser marcadamente diferentes en las Tierras Bajas noroccidentales, de modo particular en la ciudad de Palenque. Este artículo discute las ofrendas de los templos de Palenque, así como los resultados preliminares de las excavaciones realizadas en un grupo residencial y encuentra que los materiales de los depósitos rituales de este sitio a menudo comprenden posibles materiales orgánicos en lugar de aquéllos encontrados típicamente en los ejemplos de las Tierras Bajas orientales. Con el fin de considerar cómo tales prácticas eran distintas en esta región, se propone un enfoque más integral de una arqueología del ritual que incluya la presencia de microrresiduos de las acciones rituales.
\end{abstract}

Palabras clave: ritual, microarqueología, ofrendas, Palenque, Grupo IV.

ABSTRACT: Excavations throughout the eastern Maya lowlands of Mexico, Guatemala, and Belize have revealed widespread patterns in ritual practice, including patterns in the types of materials used in ritualized offerings and human burials placed within the floors and plazas of public temples and private homes. Yet, the material traces of past ritualized events appear to be markedly different in the western lowlands, particularly at the city of Palenque. This article discusses the caches recovered from Palenque's temples as well as the preliminary results of excavations carried out in a residential group and finds that the materials included in these ritual deposits often include possible organic materials rather than the typical materials found in eastern lowland examples. To consider just how practice was distinct in this region, a more comprehensive approach to an archaeology of ritual is proposed, one that includes a consideration for the micro-scale residues of ritualized actions.

KeYwords: ritual, microarchaeology, offerings, Palenque, Group IV.

RECEPCIÓN: 22 de agosto de 2017.

ACEPTACIÓN: 26 de noviembre de 2017.

DOI: 10.19130/iifl.ecm.2018.52.932. 


\section{Introducción}

Este trabajo pretende considerar una perspectiva arqueológica que emplea un enfoque metodológico de diferentes escalas para la recuperación y análisis de los materiales depositados como resultado de las prácticas rituales de los antiguos mayas. ${ }^{1}$ El enfoque aquí propuesto incluye la incorporación de técnicas microscópicas que no son empleadas por lo común en la arqueología orientada al estudio de los rituales mayas clásicos. La mayor parte de los restos arqueológicos de antiguos eventos rituales está conformada por macrorrestos, aquellos que son claramente visibles y se han conservado durante siglos. Sin embargo, algunos restos incluyen pequeñas evidencias o tan solo indicios de materiales no conservados por completo, tales como sedimentos descoloridos o restos de cenizas. Éste es el caso que aquí presentamos, en el cual intentamos comparar los depósitos rituales documentados de forma arqueológica en Palenque con aquellos pertenecientes a otros sitios del área maya. Los depósitos rituales recuperados en Palenque parecen bastante modestos en comparación con los reportados en las Tierras Bajas orientales, en sitios como Piedras Negras, Tikal, Caracol o Copán, donde se utilizaron materiales foráneos, entre ellos jade, excéntricos de piedra tallada, conchas marinas, espe de pirita, etc., correspondientes a eventos rituales y depositados como ofrendas durante todo el período Clásico (250-800 d.C.). Algunas de estas ofrendas se han recuperado de los templos de Palenque, pero los materiales son significativamente diferentes a las colecciones de materiales documentados por lo común en las estructuras de las Tierras Bajas orientales. En su lugar, los depósitos rituales de Palenque no contienen objetos visibles, pero se observan suelos con diferente coloración o con pequeños fragmentos de estuco, o se ofrendaron restos de animales y en algunos casos fósiles (Cuevas y Alvarado, 2012; García Moll, 1985; Joyce, 1992; Ruz Lhuillier, 1954, 1958). La elección de incluir lo que se sospecha eran materiales orgánicos en su mayoría puede atribuirse a una práctica distintivamente palencana. Ello podría deberse en parte a una participación diferenciada dentro de la red comercial más amplia de esos materiales tan comúnmente utilizados en la región oriente. Y al hecho de que, aunque eran partícipes de la cosmología maya en general, impulsada por una relación de reciprocidad entre los seres humanos y lo sobrenatural en donde se requerían ofrendas materiales, se atribuyó importancia ceremonial significativa a un conjunto diferente de materiales. Con el creciente número de estudios arqueológicos sumamente detallados, que incluyen el análisis de residuos químicos, análisis botánicos y de suelos, parece ser que tal escala está siendo poco considerada o de manera muy paulatina en el estudio de las prácticas rituales.

\footnotetext{
${ }^{1}$ Este artículo presenta los resultados del trabajo realizado como parte del Proyecto Regional Palenque, dirigido por el doctor Rodrigo Liendo de la Universidad Nacional Autónoma de México y financiado por una subvención UC MEXUS DI-15-7. El análisis del PEB fue realizado por el doctor Felipe Trabanino y el de la fauna fue llevado a cabo por el maestro Carlos Varela.
} 
Argumentamos que Palenque ofrece una oportunidad interesante para emplear un enfoque específico en el análisis del ámbito ritual.

Los estudios de textos e imágenes, las analogías basadas en etnografías de poblaciones mayas contemporáneas y, más recientemente, los análisis paleoetnobotánicos sugieren que diversos materiales orgánicos, como alimentos, flores, gomas, resinas y pigmentos fueron probablemente utilizados en los rituales (Bozarth y Guderjan, 2004; Brown, 2004; Morehart y Butler, 2010; Stone, 2002; Vogt, 1976). Además, estos estudios proponen que los eventos rituales a menudo fueron acompañados por bailes y representaciones con uso de disfraces, el consumo de tabaco o de bebidas y la quema de resinas de árboles como el copal (Brown, 2004; Hanks, 2013; Stone, 2002; Stuart, 1998; Tozzer, 1941). Algunas de estas acciones podrían haber producido diferentes clases de residuos, tales como restos de plantas quemadas o carbonizadas, o incluso pigmentos y materiales sin quemar que fueron parte del vestuario y pintura corporal para danzas y eventos públicos (Hutson y Terry, 2006; Houston et al., 2009; Morehart, Lentz y Prufer, 2005). Incluso contando con narraciones históricas que detallan una amplia gama de eventos rituales, en donde se incluyen acontecimientos biográficos (por ejemplo, muerte, ceremonias de curación, transición de etapas de la vida, matrimonio, nacimiento, etc.), así como otros eventos a menudo relacionados con celebraciones calendáricas o religiosas. Se cuenta también con abundante iconografía del periodo Clásico que representa una gran variedad de acciones rituales. La arqueología sólo ha recuperado evidencia de tres tipos (a menudo estrictamente definidos) de estos eventos: ofrendas (caches), entierros y ofrendas de terminación. Estas tres acciones distintas son por lo común entendidas como basadas en los principios cosmológicos mayas sobre la muerte y el renacimiento (Mock, 1998). Además, este tipo de depósitos a menudo es asumido como el resultado de un solo evento. A su vez, los arqueólogos han recuperado evidencia que muestra, al menos en entierros humanos, que hubo acciones que se repitieron, como el reingreso a las tumbas (Chase y Chase, 2011). La inclusión de estudios más detallados sobre el aspecto ritual puede ser un punto de inflexión para ampliar nuestra comprensión actual de la práctica ritual de los antiguos mayas, de manera particular en la comprensión de la temporalidad de eventos rituales desde la perspectiva del tiempo vivido, intergeneracional o de los ritmos de vida (Boivin, 2000; Tringham, 2000).

La aproximación arqueológica aquí propuesta incluye un enfoque centrado en los procesos de formación de contextos, el principio de deposición estructurada de la estratigrafía y los residuos microscópicos, a menudo dejados sin investigar, además de las estrategias tradicionales empleadas en arqueología maya para investigar la materialidad de las prácticas rituales entre los antiguos palencanos. Para ello, consideramos patrones en los depósitos rituales reportados en sitios a lo largo de las Tierras Bajas orientales, ya que son más conocidos y muestran patrones claros a través del tiempo. Para lograr estos objetivos consideramos algunos contextos de eventos rituales documentados en Palenque y lo que parece 
ser la aparición de una forma local de una práctica más amplia. Luego realizaremos una breve reseña sobre el creciente número de estudios a nivel microescala y argumentaremos sobre la necesidad de integrar de forma explícita estos métodos en los estudios sobre lo ritual. Este tipo de análisis se considera simultáneamente junto con los textos epigráficos, la iconografía, la distribución particular de restos macroscópicos, así como las descripciones etnográficas y las narraciones históricas. La combinación de tales enfoques ofrece una imagen mucho más rica de las prácticas rituales pretéritas que cualquiera de estas líneas de evidencia por sí solas. Concluimos reuniendo estos enfoques, macro y micro, considerando el marco de los trabajos recientes en el Grupo IV de Palenque y los resultados preliminares de las excavaciones realizadas en una de las estructuras al oriente de este conjunto arquitectónico.

\section{“Depósitos rituales" en las Tierras Bajas mayas}

Por más de un siglo, los arqueólogos se han fascinado con la materialmente rica vida religiosa y ritual de los antiguos mayas. Numerosas ofrendas se han encontrado en los pisos y escaleras de las estructuras de los templos. Piedra finamente tallada, monumentos de estuco y tabletas con representaciones de lo sobrenatural decoraron las estructuras de las ciudades de las Tierras Bajas. La evidencia ritual y religiosa ha sido tan frecuente y visible que algunos de los primeros estudiosos de los mayas llegaron a la conclusión de que las ciudades eran gobernadas por sacerdotes y que el centro de las ciudades fue construido solo para la adoración y el compromiso con los dioses (Becker, 1976). La investigación histórica y etnográfica continua, las excavaciones a gran escala y las traducciones jeroglíficas han cambiado desde entonces esa interpretación. Sabemos ahora, por supuesto, que los mayas clásicos constituían una civilización compleja con preocupaciones políticas, episodios de guerra, amplias redes comerciales y estructuras sociales jerárquicas en todos los niveles de la sociedad. Sin embargo, estas diversas facetas de la antigua vida maya estaban estrechamente entrelazadas, y los dioses y los antepasados, las plantas, los animales y las montañas estaban siempre presentes y tan activos como los mayas vivientes.

Arqueológicamente, las evidencias materiales más comúnes de eventos rituales entre los antiguos mayas incluyen las ofrendas (caches), los entierros humanos y los actos de "terminación". Estas acciones definidas de manera amplia caen por lo general bajo la categoría de "depósito ritual". El "depósito ritual" es reconocido como una colección de materiales resultante de acciones distintas de las actividades cotidianas. Durante el período Clásico (250-900 d.C.) estos eventos se hacían cada vez más elaborados en los templos públicos y entre la élite con materiales tales como jade y otras piedras verdes, materiales marinos, incluyendo Spondylus, punzones, corales, percebes y otras conchas tanto trabajadas como sin trabajar, obsidiana y pedernal, minerales como pirita y hematita, y materiales 
tóxicos como mercurio líquido y cinabrio. A menudo se encuentran en contextos que se consideran ofertorios o como un medio para santificar de forma intencional un espacio en que los materiales están situados espacialmente de una manera que marca un lugar particular como significativo. A medida que las excavaciones continuaron a lo largo de las Tierras Bajas y el número de ofrendas (caches) reportadas aumentó, los patrones en el tipo de materiales que se depositan de este modo, así como la ubicación y la naturaleza de su colocación, se hicieron evidentes (Freidel y Schele, 1988; Lomitola, 2012; Joyce, 1992; Maxwell, 1996; Mock, 1998). Los patrones generales de los depósitos rituales comenzaron durante el período Preclásico Tardío (400 a.C.-100 d.C.), ya que un cierto número de acontecimientos rituales parecen haber sido estructurados de acuerdo con principios cosmológicos de estratos y cardinalidad, particularmente durante la santificación de los espacios públicos ceremoniales.

Uno de los ejemplos más antiguos es la conocida "ofrenda cruciforme", hallada en el sitio de Cival en el Petén guatemalteco, en la que cuatro ollas grandes fueron colocadas en las direcciones cardinales con una quinta olla en el centro rodeada por hachas de piedra verde (Estrada-Belli, 2006). Esta ofrenda se colocó durante el período Preclásico Medio, aproximadamente en 500 a.C. Otros ejemplos tempranos incluyen aquellos encontrados en el sitio zoque de San Isidro, Chiapas, en los cuales se orientaron las orejeras de carrete de jade y las hachas de piedra verde alrededor de un plato central (Taube, 2005: 25). La construcción de templos y los eventos de deposición en ofrendas se intensifican en toda la región durante el Preclásico Tardío y el Clásico, adquiriendo el oriente una importancia simbólica particular, como se puede ver en la construcción de grupos monumentales y en el número de ofrendas que a menudo los acompañaban. Como una sociedad cada vez más agrícola, el sol era vital para el crecimiento de los cultivos. La importancia de la dirección este como el inicio del camino del sol y fuente de la regeneración era un tema que a menudo se ritualizaba a través de eventos de ofrenda, ya sea por medio de intercambios con lo sobrenatural o del entierro de los muertos (Carrasco, 1990; Joyce, 2011). La cardinalidad en general fue un importante principio cosmológico que a menudo se materializó durante los eventos rituales; esto ha sido claramente demostrado en particular en Tierras Bajas orientales.

Por ejemplo, en el sitio de Cerros (ahora Cerro Maya), en Belice, fue hallada una ofrenda de pendientes de jadeíta, tallados en forma de cabezas, en un patrón cruciforme dentro de la Estructura 6B, también fechada durante el período Preclásico Tardío (Freidel y Schele, 1988). La posición intencional en las direcciones cardinales y con significado numérico se puede ver en otras partes en las Tierras Bajas del este durante esta época. En el sitio de K'axob, Belice, se encontró una ofrenda conocida como "ofrenda triádica", que contenía una colección de objetos dispuestos en conjuntos de tres (Harrison-Buck, 2004). En las ruinas de Arenal, Belice, se colocaron dos ofrendas en la cabeza y los pies de un individuo enterrado en uno de los templos principales que incluían una colección de figuras antropomorfas (tipo "Charlie Chaplin"), así como coral, jade y "arena de playa”. 
Las figurillas estaban orientadas cardinalmente dentro de los contenedores (Taschek y Ball, 1999).

Los objetos no sólo estaban dispuestos horizontalmente, sino también de forma vertical, lo que llevó a algunos a describirlos como "cosmogramas" materializados; unos de los más elaborados fue excavado en el sitio de Caracol, Belice. A gran profundidad dentro de la estructura central del "Grupo E" de Caracol, se colocó una ofrenda que contenía una gran colección de objetos dispuestos vertical y horizontalmente hacia las direcciones cardinales (ver Chase y Chase, 2006, para una descripción completa de esta ofrenda). Dichos objetos incluían grandes conchas en bruto que encerraban una colección de pequeñas figuras antropomorfas y zoomorfas de jade, espinas de raya y un gran conjunto de orejeras de jade, además de semillas y rastros de una colmena cerca de la parte superior del depósito. Todo el conjunto estratificado se colocó en un recipiente con tapa sobre una capa de malaquita cruda dentro de un pozo y se selló con piedras. Con elementos que representaban el mundo acuático, el nivel terrestre del mundo vivo y el superior del sobrenatural, esta ofrenda agrupada era una réplica miniaturizada del cosmos maya, que fue insertada en el núcleo del templo durante un evento de santificación.

En las Tierras Bajas orientales en particular, las ofrendas también se colocaron debajo de los monumentos de piedra. En suma, los materiales que se recuperan de los depósitos rituales son duraderos y visibles. Sin embargo, muchas veces, los recipientes de cerámica con tapa que contienen estos materiales también incluyen sedimentos sueltos, que parecen ser sólo tierra. En Caracol, se han recuperado múltiples ofrendas que también incluyen colecciones sueltas de jade, desechos microscópicos de talla de concha, cinabrio y lascas sueltas de desechos líticos. A menudo denominada como la "tierra de la ofrenda" (Chase y Chase, 2012: 7), la matriz puede ser el resultado de barrer o recoger los escombros de las áreas de manufactura o de áreas usadas en la elaboración. En Kaminaljuyú, una serie de recipientes depositados labio con labio en tumbas contenían sólo cenizas, lo que los llevó a ser clasificados como cuencos de "cenizas", con la suposición de que en ellos se quemó incienso (Kidder, Jennings y Shook, 1946: 93). Mientras que algunas de las vasijas tenían evidencia de combustión en su superficie, otras simplemente contenían cenizas de "una sustancia" que quizá fue quemada en otra parte y luego depositada en el recipiente antes de ser colocada en la tumba.

\section{“Depósitos rituales" en Palenque}

Un análisis de los depósitos rituales en la ciudad maya clásica de Palenque, en la región de las Tierras Bajas noroccidentales de Chiapas, México, revela distinciones en la materialidad del ritual, en comparación con los grandes centros políticos en las Tierras Bajas centro-orientales. Mientras que en los centros políticos de Caracol, Tikal, Uaxactún y Piedras Negras frecuentemente se colocaban ofren- 
das muy elaboradas, similares en contenido y colocación, que incluían conjuntos de jade, concha, obsidiana, espinas de raya y pirita, entre otros materiales duraderos y visibles, los restos de acontecimientos rituales recuperados de Palenque son distintos en su composición material. Una consideración de los depósitos rituales, en particular aquellos dentro y alrededor de una serie de templos conocidos como el "Grupo de la Cruz", revela que las ofrendas son mucho menos elaboradas, con una preferencia por materiales en gran parte orgánicos y perecederos, llevándonos a argumentar que una tradición localizada surgió en la región de Palenque, que, aunque participaba dentro de una cosmología maya mayor, dio lugar a conjuntos rituales distintivos. La mayoría de los depósitos rituales recuperados del Grupo de la Cruz se componían de sedimentos, suelos de color y matriz cenicienta (García Moll, 1985). Una serie de ofrendas se introdujo en el suelo que rodea la sala del santuario interior del Templo de la Cruz (Figuras 1 y 2).

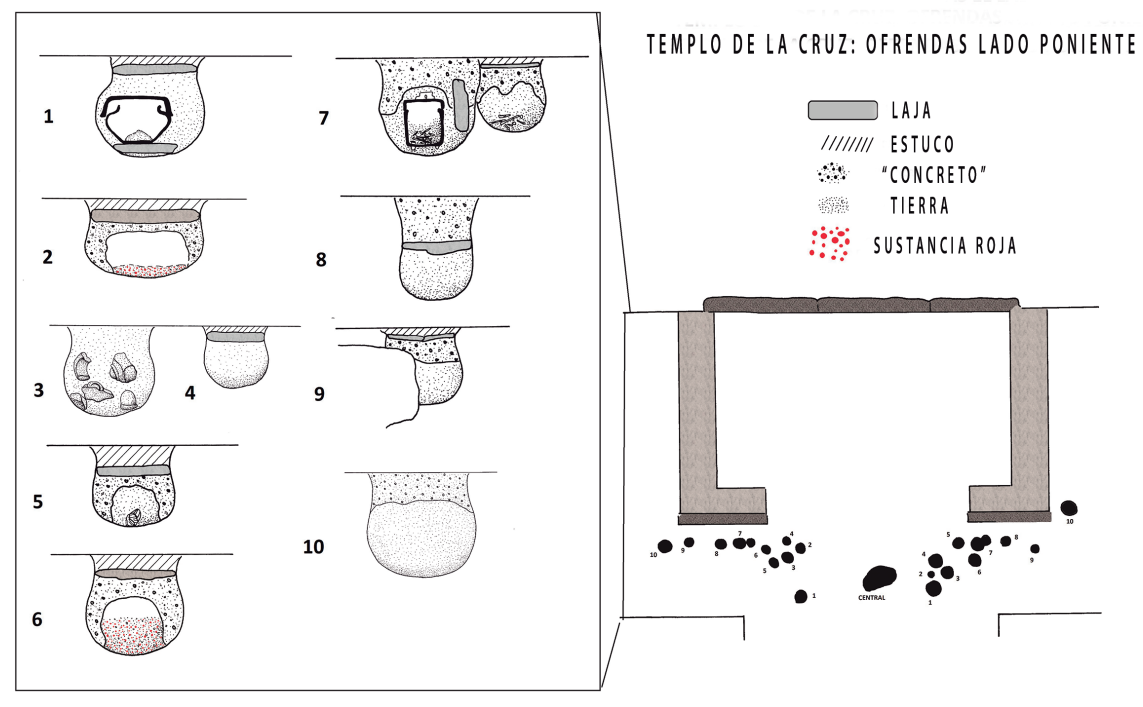

Figura 1. Templo de La Cruz, plano de ofrendas, lado poniente. Tomado y modificado de García Moll (1985).

Los pozos fueron bien documentados por los excavadores originales, incluyendo las descripciones de textura y color de los sedimentos. La Ofrenda 1, en el lado occidental de la habitación, era un solo recipiente con tapa que contenía "tierra blancuzca", el pozo entonces fue rellenado con "tierra", tapado con una gran laja de piedra y luego sellado con el piso de estuco. ¿Eran las cenizas de una sustancia quemada in situ? ¿La tierra fue transportada de otra parte para sellar la ofrenda dentro del templo? El pozo de la Ofrenda 2, al lado de la primera 


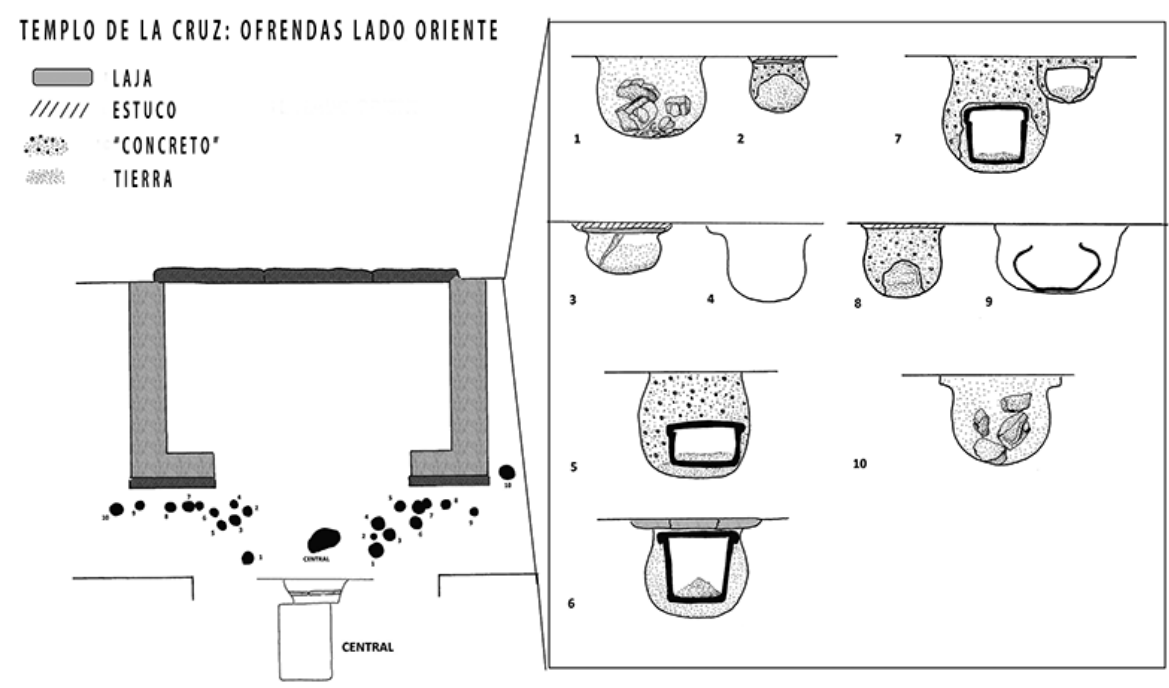

Figura 2. Templo de La Cruz, plano de ofrendas, lado oriente. Tomado y modificado de García Moll (1985).

ofrenda, contenía solo una "sustancia roja”. La sustancia que se encontró quizás era cinabrio, usado con frecuencia en rituales mayas antiguos. ¿Pero ese cinabrio originalmente cubría algo más, algo perecedero como un objeto de madera?, ¿o simplemente se esparció en el fondo del pozo? También la Ofrenda 2 "consistía en un objeto de forma caprichosa, probablemente de alguna sustancia orgánica que con el tiempo se desintegró, pero que dejó grabada en el concreto de cal con el que fue cubierto, su forma primitiva” (García Moll, 1985: 206). Una serie de las ofrendas parece estar cubierta por "concreto.” ¿Es posible que esta capa distinta de la matriz se obtuviese con el propósito de cubrir y sellar la ofrenda dentro del pozo? De hecho, sólo unos pocos materiales encontrados en los pozos intrusivos en el piso del Templo de la Cruz eran visibles e identificables de manera clara, entre los que se incluyen vasijas cerámicas, varios huesos de fauna, fragmentos de fachadas de estuco y, en un caso, un fragmento de núcleo de obsidiana. Sin embargo, 10 de los 21 pozos tenían objetos visibles y contenían sólo suelo ceniciento, rojo o sedimentos descoloridos. Seis de las ofrendas incluyeron una vasija cerámica y todos menos uno (como la Ofrenda 7 en el lado oriente) "tierra blancuzca" (como la Ofrenda 1 en el lado poniente).

Aunque la ofrenda pudo haber sido un solo momento de deposición, las costumbres o protocolos que se siguieron en cómo, dónde y qué tipo de materiales eran aceptables para ofrendar eran resultado de una larga historia de prácticas repetitivas. Las ceremonias de construcción de viviendas se observan todavía entre las comunidades mayas contemporáneas, en ellas una casa de nueva construcción es "alimentada" por ofrendas intrusivas en el piso, muchas de las cuales son 
perecederas, como pollos, sangre, licor e incienso (Vogt, 1976: 52). Del mismo modo, los templos antiguos fueron "alimentados" con ofrendas colocadas en los pisos. Los "depósitos rituales" encontrados por los arqueólogos son el resultado de la historia del involucramiento de éstos con los materiales, lo que lleva a que se enfaticen materiales particulares a través de la ritualización. Para comprender mejor la historia de la práctica, es importante identificar el tipo de materiales incluidos y la forma en que fueron organizados espacialmente, y si se depositaron de manera intencional o no.

\section{Detección de rastros de actos rituales}

Mi interés en la materialización de los eventos rituales viene de la comprensión de que el ritual es una práctica material, que se hace significativa a través de la repetición y la experiencia (Joyce, 2011; Swenson, 2015). Una aproximación al ritual centrada en la acción y la materialidad se considera aquí como una alternativa a la comprensión problemática de la religión como un fenómeno transcultural (para un argumento alternativo véase Fogelin, 2007). Las evidencias materiales del ritual a menudo se modelan a través de la práctica repetitiva, realizada de maneras particulares, con la inclusión de materiales específicos y en espacios prescritos. Este patrón en las evidencias materiales forma una "deposición estructurada" (Joyce, 2008; Pollard, 2010; Richards y Thomas, 1984). Los patrones de distribución de artefactos a través del espacio y del tiempo se asumen a menudo (no siempre) como el resultado de actos más o menos "intencionales". Tales prácticas repetitivas pueden analizarse para entender cómo se reproduce, mantiene o cambia el ritual.

Yo sigo a otros al considerar el ritual, no como una categoría separada de comportamiento o como opuesta a la acción cotidiana, sino más bien como una forma estilizada de actuar que sirve para enfatizar aquellos componentes de lo cotidiano (Bell, 1992; Bradley, 2003; Humphrey y Laidlaw, 1994; Joyce, 2011). Un acercamiento arqueológico comprensivo a una arqueología del ritual intenta examinar las maneras en que los materiales, la gente, los lugares y las prácticas se acentúan, reconociendo que esas acciones estilizadas varían de cultura a cultura, a través del tiempo y del espacio. Para identificar un evento particular como "estilizado", se debe entender qué acciones no estilizadas, o cotidianas, se practican en esa cultura particular. A menudo, la evidencia de las acciones cotidianas se puede encontrar en capas muy finamente estratificadas, a través del pisoteo de la gente, el procesamiento de comida, el dormir en esteras, el vertido de basura orgánica, etc. Y como proponemos aquí, esas capas estratificadas son también claves para entender las acciones rituales, en especial si esas acciones incluyen el uso de materiales orgánicos y en gran parte perecederos. Es por ello que resulta necesario muestrear ambas áreas, tanto de presuntas acciones rituales y de actividades no-rituales. 
Una arqueología del ritual maya preocupada con la acción, la práctica y la materialidad no ignora por completo el significado o el simbolismo, sino que permite consideraciones adicionales para explorar las muchas facetas de la ritualización y la materialización. Sin embargo, incluso la creación de significado es un proceso activo, que surge a través de la interacción material. Este enfoque orientado a la acción del ritual pone de manifiesto la variabilidad y la fluidez en la materialidad de los actos rituales. Si bien puede haber algunos principios estructurales básicos en la práctica maya clásica de deposición de ofrendas, entierros u otros eventos ceremoniales, la forma en que dichos eventos se materializaron podría variar. Algunos eruditos han reconocido los antiguos depósitos rituales mayas, particularmente las ofrendas intencionales en estratos, como la materialización de una cosmología maya, una referencia simbólica a los niveles del universo y a las cuatro direcciones cardinales del plano terrenal (Chase, 1988; Joyce, 1992). Dependiendo de cómo un investigador excave y muestree el depósito, es posible que se pierda tal estratificación intencional. Los investigadores sensibles a estos "depósitos estructurados" han identificado arreglos complejos de materiales, no como subproductos accidentales de las actividades humanas, sino como depósitos intencionalmente colocados y a menudo producto de rituales significativos (Joyce, 2008; Joyce y Pollard 2010; Pollard, 2001).

Los depósitos estructurados tienden a pasarse por alto, ya que pueden contener objetos rotos y fragmentados. Mientras que uno podría asumir que los objetos rotos son un rasgo característico de los depósitos, simplemente arrojados en su lugar de descanso final, deben considerarse, sin embargo, las relaciones materiales y los itinerarios de los objetos que revelan una compleja historia de transformación, movimiento, uso y deposición (Joyce y Gillespie, 2015). A lo largo del camino, a medida que los materiales y la gente se reúnen, los valores y el significado emergen, cambian, de modo que incluso el fragmento de un objeto creado una vez o de una forma considerada "entera" mantiene parte de su valor anterior, memoria y significado. El itinerario de un material, sus características físicas y cualidades estéticas, los recuerdos y el significado que invoca, cualquiera de éstas o todas sus características juntas pueden conducir a su ritualización. Es por tal razón que los materiales que participaron en los antiguos rituales mayas son a menudo muy variables y poseen diversos estados de "completitud".

\section{Una microarqueología del ritual}

En este trabajo intentamos detectar capas finamente estratificadas, que por lo general no se ven a simple vista. Para ello propongo la inclusión de tipos de análisis múltiples con el fin de recuperar la evidencia material potencial de eventos deposicionales singulares y la estratificación a través del tiempo, cuyo espacio particular se santifica y se mantiene a través de repetidos eventos rituales. La micromorfología, en conjunto con el análisis de las muestras de suelo no pertur- 
bado en bloque, cortado en secciones delgadas y visto microscópicamente, es un método ideal para dicho propósito. Con este tipo de análisis podemos ver los componentes presentes en una matriz y cómo están relacionados espacialmente. Podemos buscar arreglos de microartefactos y ecofactos. Las capas compactadas o las capas de materiales con dibujos pueden indicar una deposición intencional, resultado de la actividad humana, o pueden revelar períodos de abandono o exposición. Por ejemplo, el análisis micromorfológico llevado a cabo en una cueva sudafricana detectó múltiples capas finas de combustión entre el limo arrojado por el viento, lo que indicaba que lo que visiblemente parecía ser sólo un episodio era de hecho una serie de eventos de combustión con períodos de inactividad humana entre ellos (Goldberg et al., 2009). En otro estudio realizado en el suroeste de los Estados Unidos se utilizó micromorfología para analizar la secuencia compleja de eventos que condujeron a la terminación ritual de las estructuras de kiva (Van Keuren y Roos, 2013). Lo que encontraron fue la presencia de sedimentos no locales a la zona depositados en una gruesa capa a través del suelo y el banco de la estructura antes de su destrucción final. Una serie de eventos de quema fue detectada durante la destrucción del edificio. Por último, las capas de viento y el sedimento desgastado por encima de tales episodios señalan el abandono final. Este ejemplo particular es muy relevante para el área maya donde los eventos de "terminación" son a menudo citados como la causa de las capas macroscópicas de quemar y romper objetos. Sin embargo, ¿fue la "terminación" el resultado de una serie de acciones ininterrumpidas o la "terminación" ocurrió a través de etapas separadas de acciones, cada una de ellas puntuada por períodos de inactividad?

El evento ritual es temporal. Es un momento elaborado en una serie de momentos. Debido a su elaboración, crea memoria, la cual se comparte entre aquellos que participaron activamente y fueron testigos del evento. Los recuerdos pueden ser rememorados y compartidos entre aquellos que no fueron testigos ni participaron. A través de generaciones de eventos rituales esos recuerdos se convierten en tradición, un conjunto de prácticas que se enseñan y aprenden, alterados por momentos de creatividad. Una arqueología preocupada por la temporalidad de la ritualización, la construcción de la tradición y el materialismo activo de la memoria social necesita las herramientas necesarias para recuperar la evidencia de la repetición y las distinciones en la materialización de tal evento.

La micromorfología no sólo se ocupa del uso del espacio horizontalmente, sino que puede revelar la temporalidad de esas actividades en la alteración que se produce con el tiempo. Un número de arqueólogos se han alejado de considerar el "registro material" como bloques estáticos de tiempo, pues buscan entender el ritmo fluido del tiempo vivido. Las interpretaciones de acontecimientos pasados pueden ser resituadas como los momentos vividos por generaciones, conduciendo a siglos de evidencias materiales que son alteradas, excavadas o pisoteadas. A menudo estas huellas ocurren involuntariamente mientras que los seres humanos, las plantas y los animales apenas se están moviendo alrededor 
del paisaje, realizando actividades relacionadas con la vida cotidiana. En otros casos, tales evidencias se producen a través de acciones intencionales, que alteran el espacio. Por ejemplo, un estudio etnoarqueológico llevado a cabo por Nicole Boivin (2000) encontró que, durante los eventos señalados, como los eventos del ciclo de vida, o durante las ocasiones de culto anual, los pisos de una casa rural indígena fueron recubiertos y coloreados. Tomó muestras de micromorfología de esos pisos y las comparó con muestras que recuperó del sitio arqueológico de Çatalhöyük, Turquía, y encontró un patrón similar en la estratificación y tipo de los componentes, lo que sugiere que los pasados ocupantes de Çatalhöyük también recubrieron repetidamente sus paredes a un nivel bastante regular en intervalos. Aunque sabemos que las áreas habitadas y utilizadas habitualmente se limpian con regularidad, a menudo son los rastros microscópicos los que permanecen como evidencia de la actividad in situ.

Al considerar los resultados de los estudios micromorfológicos realizados en otros lugares se puede argumentar que las estructuras construidas en piedra y las superficies estucadas de las plazas del antiguo paisaje maya proporcionarán unidades bien definidas para el análisis de las secuencias deposicionales. La excavación con la técnica de la Matriz de Harris permite un mapeo complejo de las relaciones espaciales entre las características intencionalmente construidas y las áreas de actividad visibles. La integración de un análisis a nivel microscópico podría revelar potencialmente huellas invisibles de materiales orgánicos en el tejido mismo de estas áreas. Tales técnicas se aplican en contextos considerados rituales y en otros que no lo son; este tipo de enfoque microscópico permite la comparación de rasgos materiales que resultan en acciones distintivas y elaboradas. Un esquema de muestreo similar fue empleado en un estudio desarrollado por Matthews et al. (1997) en el Cercano Oriente, en el cual compararon secciones delgadas recuperadas de varias áreas de actividad y encontraron que las áreas como altares y entierros contrastaban con las de actividades domésticas. Alrededor del altar, los pisos estaban finamente enyesados, más limpios (a diferencia de las gruesas capas para transitar) y, a veces, incluían materiales quemados y ocre.

La micromorfología combinada con los análisis de suelos sueltos es mucho más eficaz para identificar no sólo cómo los componentes están estratificados y espacialmente relacionados con el tiempo, sino qué tipo de materiales y, potencialmente, qué cantidad, fueron incluidos. Minerales, huesos, microartefactos, carbono, entre otros se pueden detectar a través de la micromorfología, pero dependiendo de la ubicación en el bloque desde el que se cortó la sección delgada; una vista plana de los componentes hace que los componentes particulares, tales como los fitolitos, sean difíciles de identificar. A veces, los fitolitos se detectan en láminas micromorfológicas; sin embargo, los análisis de suelos sueltos, utilizando un proceso de extracción química, son mucho más adecuados para identificar especies de plantas. Se realizaron análisis paleoetnobotánicos, en particular el análisis fitológico, sobre una serie de vasijas ofrendadas, colocadas labio a 
labio, en el sitio clásico maya de Black Creek, Belice, que revelaron la presencia de maíz, calabaza, frutas, flores y esponjas marinas (Bozarth y Guderjan, 2004). La iconografía, el texto, los relatos históricos y la etnografía confirman que los alimentos, las flores, el copal, las resinas y el caucho se inclú́an a menudo en eventos rituales como ofrendas, vestimentas de altar y festejos para participantes rituales (Tozzer, 1941; Vogt, 1976; Stone, 2002). Sin embargo, pocos estudios han buscado recuperar de forma explícita las evidencias materiales de estas materias, concentrándose en cambio en los objetos visibles y fácilmente recuperables. Ello se debe en especial a la fiabilidad de los métodos disponibles, pero este tipo de técnicas de microescala han mejorado drásticamente en las últimas décadas y ahora se han convertido en lugar común en los estudios arqueológicos.

A través de una colaboración con el Proyecto Regional Palenque (Liendo, 2013), un equipo especializado de investigadores recientemente tuvo la oportunidad de emplear estas técnicas en la excavación de un grupo residencial de Palenque. Nuestro objetivo era investigar y recuperar las capas de deposición atribuidas a los eventos a nivel de la casa, en la atmósfera política mayor de Palenque y en la región maya más amplia. A través del texto jeroglífico disponible recuperado del grupo residencial y de las fechas de carbono asociadas con episodios de deposición y construcción, podemos considerar las generaciones de tiempo vivido en el Grupo IV, como el hogar de Chak Sutz'.

\section{Grupo IV}

El Grupo IV es una casa de élite situada a unos 300 metros del palacio principal de Palenque (Figura 3). El grupo de plazuela fue sometido a una investigación arqueológica preliminar durante los años cincuenta después del descubrimiento accidental del grupo durante la construcción de la carretera principal en el sitio (Ruz Lhuillier, 1952). Estas primeras exploraciones recuperaron un tablero tallado, conocido como el Tablero de los Esclavos. El panel mayor está inscrito con un texto que identifica a Chak Sutz', un señor subordinado o sajal del gobernante K'inich Ahkal Mo' Nahb, dato utilizado por los principales investigadores para argumentar que el Grupo IV debía ser la residencia de dicho señor menor (Josserand, 1991; Schele, 1986; Stuart y Stuart, 2008). El tablero inscrito, que fue colocado en la gran estructura de dos pisos del lado occidental de la plaza, muestra una escena tallada que representa al rey, K'inich Ahkal Mo' Nahb, recibiendo las insignias de entronización de sus padres e incluye textos que describen momentos significativos en la vida de un importante jefe de la casa, Chak Sutz' (Josserand, 1991). El texto identifica a Chak Sutz', como un líder militar, un señor bajo las órdenes de K'inich Ahkal Mo' Nahb, y narra los logros de la vida de Chak Sutz' durante el siglo VIII temprano, indicando con ello que esta casa, y el grupo de gente que allí vivía, tenía una relación con la familia gobernante. Ellos eran claramente de una clase noble. Y así, al tomar en cuenta la serie de eventos que 
ocurrieron en el Grupo IV y la evidencia material de los actos rituales, también consideramos el paisaje político mayor de Palenque.

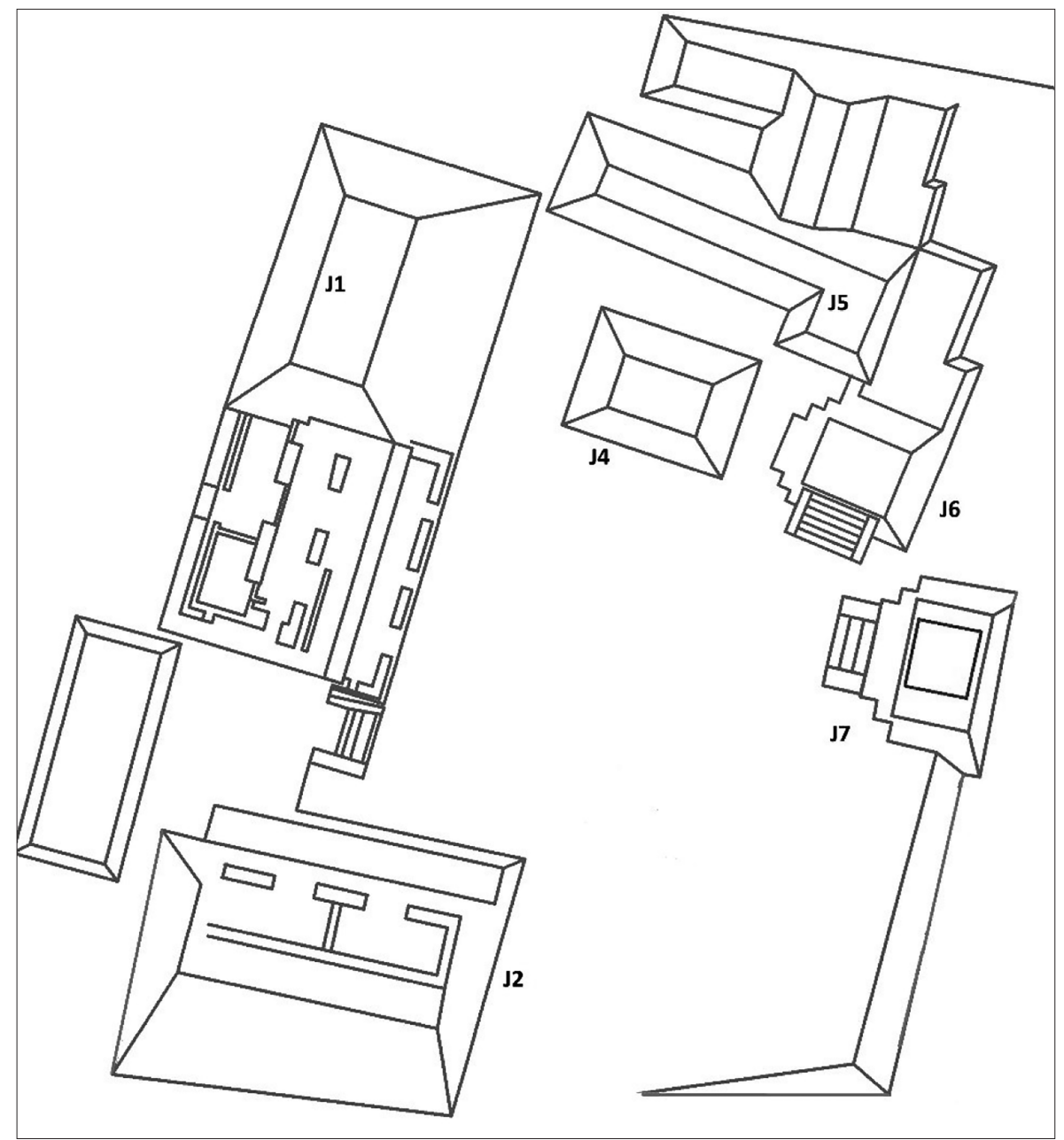

Figura 3. Plano del Grupo IV. Dibujo de Campiani (2016).

Este grupo residencial en particular es aún más interesante, ya que las investigaciones posteriores identificaron una concentración de entierros en el lado oriental de la plaza (Rands y Rands, 1961). Rands y Rands recuperaron un total de 13 entierros, algunos de los cuales se colocaron uno encima del otro, desde la roca madre, indicando una larga historia que abarca varias generaciones de even- 
tos de enterramiento. Raramente se han recuperado tales grupos de entierros en la región maya, lo que llevó a Rands a describirlo como un "cementerio" (Rands y Rands, 1961). Lo que está claro es una preferencia por la dirección este en los eventos ceremoniales llevados a cabo en el Grupo IV. Proyectos posteriores dirigidos por arqueólogos del Instituto Nacional de Antropología e Historia durante los años noventa también reconocieron este foco ceremonial orientado hacia el este, lo que provocó nuevas excavaciones, que resultaron en la recuperación de cinco entierros adicionales en la base de las Estructuras J6 y J7 (López Bravo, 1995; 2004).

Más recientemente, se realizaron excavaciones en el Grupo IV para definir y reconstruir la compleja serie de modificaciones de la plaza que acompañaron a los sucesivos entierros, además de la excavación de la Estructura J7, el edificio oriental del grupo. Mientras que algunos análisis todavía están pendientes, en particular los microanálisis, como la micromorfología o los análisis paleoetnobotánicos, el objetivo era instituir una técnica de excavación de grano fino que fuera sensitiva a la práctica repetitiva, la estratificación de la actividad con el tiempo y la articulación de secuencias microestratigráficas dentro de los contextos arqueológicos más grandes y visibles, en conjunto con aquellas otras líneas de evidencia como los textos, la iconografía, la etnografía, etcétera.

\section{Estructura J7, un mausoleo oriental}

Los ocupantes del Grupo IV cortaron el estrato ocupacional más temprano para construir una cripta formal forrada de piedra para lo que pudo haber sido uno de los primeros fundadores del linaje, convertido en antepasado (Figura 4). La cripta fue construida con dos nichos en el lado oriental y occidental, lo cual resultó en un diseño cruciforme. Se han encontrado enterradas construcciones similares, con nichos construidos, en otras áreas de Palenque, como el Grupo A (Blom, 1923: figura 117) o una tumba en el Grupo Norte (San Román Martín, 2005). Toda la construcción fue luego estucada antes de colocar con cuidado a un solo hombre de 25 a 35 años. El individuo llevaba orejeras y un collar con tres cuentas de jade. Se encontró un malacate de piedra caliza tallada directamente debajo de la pelvis. Una única vasija, que contenía lo que parecía ser sólo tierra, fue colocada como ofrenda en el nicho occidental. Como la vasija estaba hecha pedazos, se tomaron muestras de los tiestos para someterlos a análisis de fitolitos con el fin de identificar potenciales restos de plantas, además de muestras que fueron sometidas a análisis químicos (cuyos resultados están pendientes). También se recuperaron muestras sistemáticas de sedimentos sueltos a lo largo de la tumba bajo el cuerpo para realizar análisis químicos. No fue posible recuperar muestras de suelo en bloque para micromorfología en este contexto, ya que la matriz en la que descansaba el cuerpo era delgada y estaba directamente encima de las lajas calizas cubiertas de estuco, colocada como un piso sobre la roca madre. 


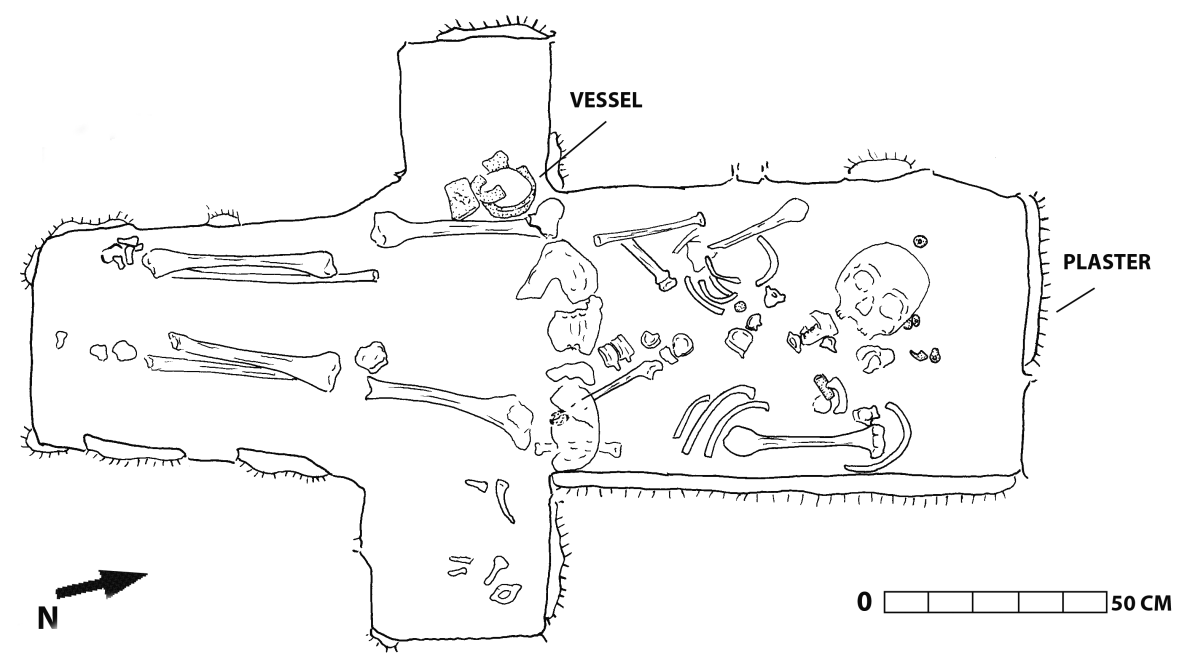

Figura 4. Plano del entierro central de la Estructura J7. Dibujo de la autora.

Las fechas de radiocarbono indican que poco después de sellar el entierro con una serie de lajas, fue construido un altar grande y cuadrado directamente encima de este individuo, aunque resulta difícil decir cuánto tiempo en meses o años. Sin embargo, las muestras de micromorfología revelan la existencia de un evento entre el sellado del entierro y la construcción del altar. La presencia de carbón y estratos de microartefactos en un suelo muy poblado revela una superficie de plaza habitada. La muestra de micromorfología tomada sobre la superficie de la plaza reveló una capa de material rocoso rico en arcilla depositado antes de la construcción del altar de piedra. Esta capa era estéril y sin artefactos. Por cerca de una generación (unos 60 años), el altar estuvo en el lado oriental de la plaza como un lugar en el que los vivos podían visitar y rendir culto a su antepasado colocado debajo (Figura 5). Se encontraron áreas concentradas de combustión en la base del altar y en la parte superior del altar. El área quemada fuera del altar fue extensamente muestreada para la datación por radiocarbono, estudios paleoetnobotánicos o PEB y análisis químicos. Los macroartefactos en este contexto incluyen un vaso de incensario y los restos de cuatro peces: una mojarra, dos tenguayacas y un pejelagarto. ${ }^{2}$ Además, había restos de tortugas y conchas de xute quemadas in situ. Los resultados preliminares indican que el pino estuvo presente en este evento de quema. ${ }^{3}$ Dicha especie no es local de Palenque, por lo que debió obtenerse fuera de la ciudad. Los estudios botánicos llevados a cabo en contextos rituales en otras partes de la región maya a menudo han re-

${ }^{2}$ C. Varela, comunicación personal.

${ }^{3}$ F. Trabanino, comunicación personal. 
cuperado restos de dicha conífera, y en esos casos se trataba de un recurso no local. El pino estuvo presente en eventos rituales realizados en cuevas de Belice (Morehart, Lentz y Prufer, 2005), y en contextos rituales del sitio de Xunantunich, Belice (Lentz et al., 2005), así como en una de las ofrendas más tempranas y elaboradas de Caracol, Belice (Chase y Chase, 2006). En cada caso, se obtuvo de otros lugares para la ocasión. Incluso hoy en día, los mayas contemporáneos a menudo incluyen ramas y agujas de pino en sus altares domésticos y celebraciones. Los relatos etnográficos describen la cama de acículas colocada alrededor del altar como una barrera protectora entre el especialista ritual y el monstruo de la tierra (Vogt, 1976).

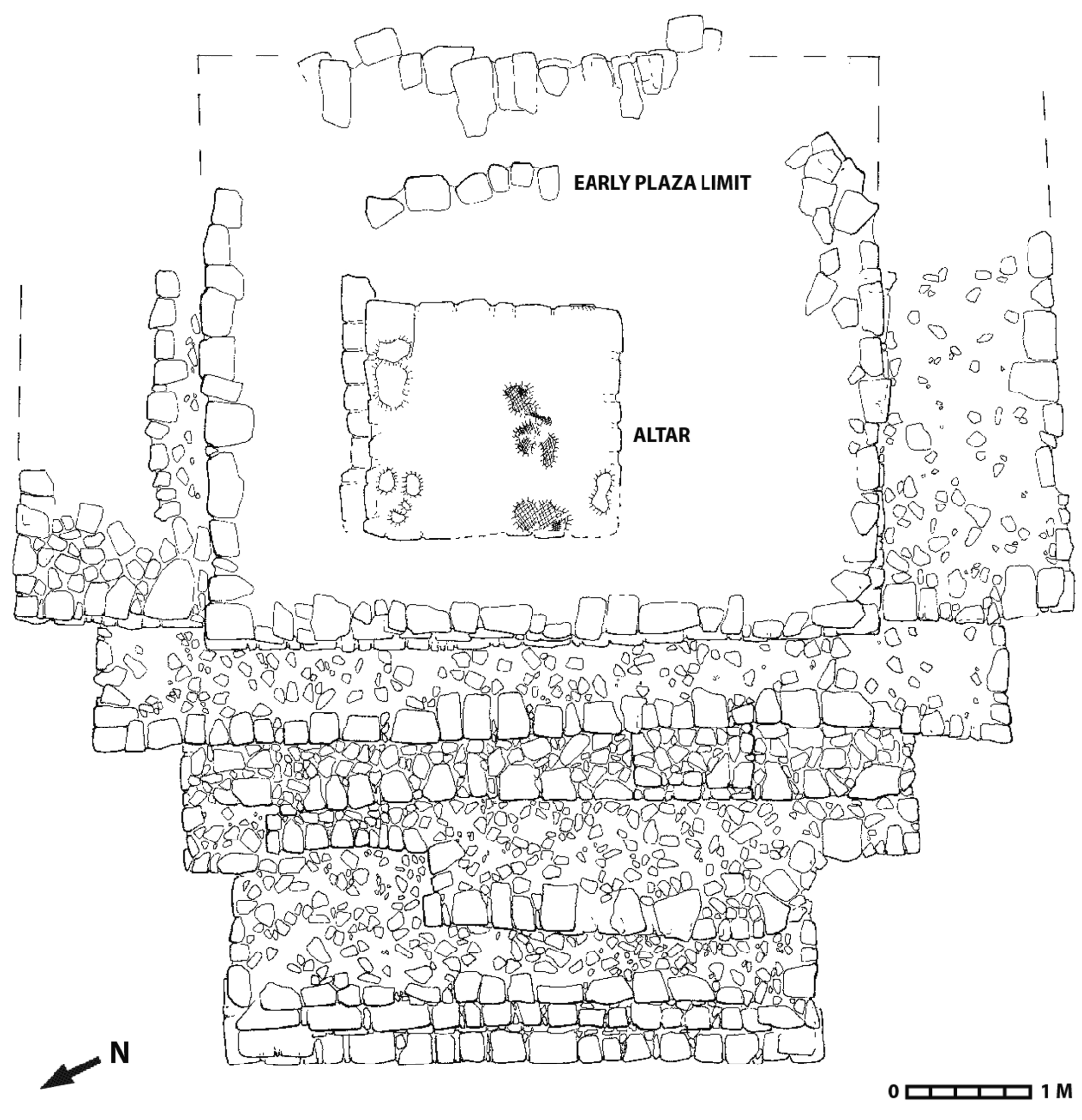

Figura 5. Plano ilustrativo de la Estructura J7. Dibujo de la autora. 
Las muestras sueltas fueron recuperadas de la superficie estucada del altar, particularmente en el área concentrada de la combustión, para el PEB y la datación por radiocarbono. Las muestras sistemáticas se obtuvieron a lo largo de la superficie para los análisis químicos. El análisis PEB preliminar sugiere que algunas ofrendas de ceiba fueron quemadas en la superficie del altar (Cuadro 1). El árbol de la ceiba era sagrado para los antiguos mayas, y a menudo fue representado en monumentos de piedra y vasos policromos (Zidar y Elisens, 2009). Con una metodología intensiva, nuestra meta radicó en recuperar la evidencia material de aquellas acciones que a menudo vemos representadas.

\begin{tabular}{|c|c|c|c|}
\hline Muestra & Contexto & C14 & $\begin{array}{c}\text { Fecha en los años } \\
\text { calendarios }\end{array}$ \\
\hline PREP400-09-11 & $\begin{array}{c}\text { Evento de quemado } \\
\text { en el relleno de } \\
\text { construcción }\end{array}$ & $1353 \pm 27$ b.p. & $\begin{array}{c}\text { Calibrado a AD } \\
636-710 \\
(91.6 \% \text { probabilidad })\end{array}$ \\
\hline PREP400-20-31 & $\begin{array}{c}\text { Quemado en la } \\
\text { superficie del altar }\end{array}$ & $1433 \pm 23$ b.p. & $\begin{array}{c}\text { Calibrado a AD } \\
583-654 \\
(95.4 \% \text { probabilidad })\end{array}$ \\
\hline PREP400-44-44 & $\begin{array}{c}\text { Entierro bajo el altar } \\
\text { Calibrado a AD } \\
576-651\end{array}$ \\
\hline PREP400-47-49 & $\begin{array}{c}\text { Directmente sobre } \\
\text { roca madre }\end{array}$ & $1441 \pm 24$ b.p. & $\begin{array}{c}95.4 \% \text { probabilidad }) \\
\text { Calibrado a AD } \\
534-616\end{array}$ \\
\hline
\end{tabular}

Cuadro 1. Fechas de radiocarbono de la Estructura J7 del Grupo IV, Palenque.

Además, se tomaron muestras de suelo en forma de bloques en la superficie y la base del altar para estudios de micromorfología. La combinación de técnicas analíticas permitirá la identificación de los componentes de la matriz, el tipo de materiales presentes y, en el caso de la micromorfología, su disposición espacial a medida que se establece en el tiempo. La datación por radiocarbono ha confirmado hasta el momento que el altar estuvo expuesto y en uso por una generación al menos. Visible a la escala macroscópica, existe un área concentrada de quema en el altar, y suponemos que sería el resultado de un evento. Sin embargo, los análisis microscópicos pueden revelar continuas y repetidas quemas durante eventos distintivos de veneración y ofrenda.

Después de casi 60 años de uso, la siguiente generación construyó un edificio cuadrado alrededor del altar, enterrándolo de forma definitiva dentro del núcleo de la estructura (Figura 6). Antes de que se terminara dicha estructura, se llevaron a cabo múltiples quemas directamente en el relleno de construcción. Estas áreas concentradas de combustión en el relleno también se muestrearon para su datación por radiocarbono y análisis de PEB. Los resultados preliminares sugieren 
la presencia de pimienta y pino, dos sustancias que producen un olor distintivo. El Grupo IV presentaba una estructura formal de mausoleo, siguiendo otros grupos residenciales identificados en Palenque, como los Grupos B y C (López Bravo, 2004). Este arreglo arquitectónico sería similar al de otras ciudades mayas clásicas, tales como Caracol y Tikal, conocido con el nombre de Plan de Plaza 2 (Becker y Jones, 1999).

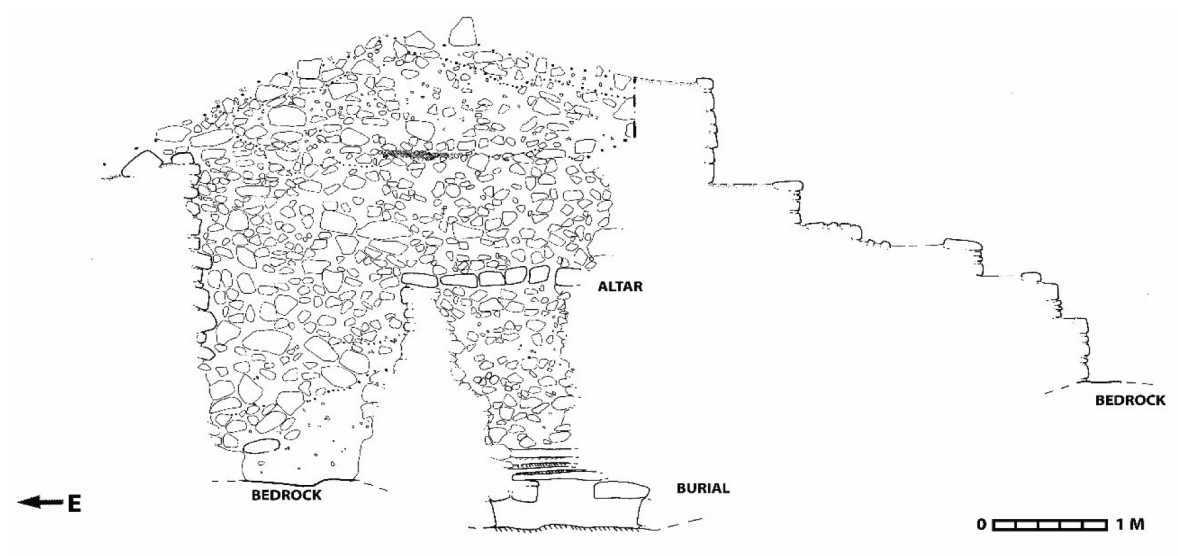

Figura 6. Ilustración de la Estructura J7 con el entierro y el altar seguidos por múltiples eventos de quemado. Dibujo de la autora.

\section{Conclusiones}

La narración que se inscribió en el monumento de Chak Sutz' describe, en más de una ocasión, un "bulto humeante de la sucesión" (Josserand, 1991: 28). El humo y la quema de sustancias, como el incienso, los alimentos, las resinas o el caucho, acompañaba una serie de eventos rituales entre los mayas antiguos, y aún entre los contemporáneos. La gama de rituales representados en la ciudad de Palenque, ya sea conmemorando a una deidad u honrando un antepasado, a menudo incluía materiales orgánicos. Las ofrendas al interior de los templos de Palenque fueron recuperadas hace décadas, cuando los métodos aquí propuestos eran inexistentes o estaban en sus inicios. Aprovechando las técnicas analíticas modernas, las excavaciones del Grupo IV han demostrado que, como parte de las ceremonias residenciales, la combustión y el humo de diversas sustancias era una característica predominante de los acontecimientos rituales. Los relatos etnográficos del ritual y de las ceremonias de sanación entre los mayas contemporáneos describen la quema como medio para dedicar ofrendas. A través del fuego, los materiales se transforman en humo, elevándose a otros mundos donde reside lo sobrenatural (Brown, 2004; Vogt, 1976). El personaje enterrado 
profundamente dentro de la estructura del Grupo IV de Palenque, bajo el altar temprano, era claramente un antepasado importante de la casa de Chak Sutz'. Durante el período Clásico Tardío, en el momento en que las estructuras con un mausoleo al este fueron más frecuentes en las tierras bajas, allí se construyó una estructura oriental más formal, de acuerdo con la tradición maya más amplia.

Stuart (1998) ha argumentado que el fuego y la quema de incienso, tal como se describe en el texto clásico maya, era un componente común de las ceremonias de renovación de las casas. La combustión que se produjo directamente en la superficie del altar, así como el incienso quemado y los restos de pescado en la base del altar, fueron destinados a "alimentar" al antepasado colocado debajo. El trabajo etnoarqueológico reciente ha revelado que éste es el caso entre los grupos mayas contemporáneos en los que la quema de materiales constituye un medio para alimentar a los dioses:

For Maya ritual practitioners, the act of burning is key to rites. It transforms the copal incense, blood, chocolate, chili, tallow, rum, sweet bread, or tobacco into smoke, thereby rendering it into a state appropriate for consumption by the deities. Thus, by burning offerings, ritual participants "feed" the deities, offering them aromatic items and enticing them into a relationship based on ritual reciprocity and commensality in this site of exchange ${ }^{4}$ (Brown, 2004: 37).

Una arqueología relacionada con la materialización de los eventos rituales preguntaría ¿qué estaban quemando? y icon qué frecuencia lo quemaban? La excavación arqueológica macroscópica tradicional no sería adecuada para responder a esas preguntas. La quema que ocurrió dentro del relleno durante la construcción del edificio posterior fue, como sugirió Stuart, un acto para alimentar a la nueva construcción con el fin de albergar en su interior al antepasado. La investigación realizada en la Estructura J7 del mausoleo oriental del Grupo IV es un buen ejemplo de la época en que la epigrafía, los textos, la etnografía y las evidencias materiales recuperadas a través de la arqueología se unen para proporcionar una comprensión más completa del evento ritual.

\footnotetext{
${ }^{4}$ Para los practicantes de rituales mayas, el acto de quemar es la clave de los ritos. Transforma el incienso del copal, la sangre, el chocolate, el chile, la cera, el ron, el pan dulce o el tabaco en humo, convirtiéndolos así en un estado apropiado para el consumo de las deidades. Por lo tanto, al quemar ofrendas, los participantes en el ritual "alimentan" a las divinidades, ofreciéndoles elementos aromáticos y atrayéndolos hacia una relación basada en la reciprocidad y la comensalidad ritual en este lugar de intercambio. (Traducción de la autora)
} 


\section{Bibliografía}

Becker, Marshall J.

1976 "Priests, Peasants, and Ceremonial Centers: The Intellectual History of a Model”, Maya Archaeology and Ethnohistory, pp. 3-20, Norman Hammond y

Gordon R. Willey (eds.). Austin: University of Texas Press.

Becker, Marshall J. y Christopher Jones

1999 Excavations in Residential Areas of Tikal-Groups with Shrines: Tikal Report 21, vol. 104. Philadelphia: University of Pennsylvania Museum of Archaeology.

Bell, Catherine

1992 Ritual Theory, Ritual Practice. Oxford: Oxford University Press.

Blom, Franz

1923 Las Ruinas de Palenque: Xupá y Finca Encanto. México: Instituto Nacional de Antropología e Historia.

Boivin, Nicole

2000 "Life Rhythms and Floor Sequences: Excavating Time in Rural Rajasthan and Neolithic Catalhoyuk". World Archaeology, 31: 367-388.

Bozarth, Steven y Thomas H. Guderjan

2004 "Biosilicate Analysis of Residue in Maya Dedicatory Cache Vessels from Blue Creek, Belize”, Journal of Archaeological Science, 31 (2): 205-215. DOI: 10.1016/j.jas.2003.08.002.

Bradley, Richard

2003 "A Life Less Ordinary: The Ritualization of the Domestic Sphere in Later Prehistoric Europe", Cambridge Archaeological Journal, 13 (1): 5-23. DOI: 10.1017/ S0959774303000015.

Brown, Linda A.

2004 "Dangerous Places and Wild Spaces: Creating Meaning with Materials and Space at Contemporary Maya Shrines on El Duende Mountain", Journal of Archaeological Method and Theory, 11 (1): 31-58. DOI: 1072-5369/04/0300-0031/0.

Carrasco, David

1990 Religions of Mesoamerica: Cosmovision and Ceremonial Centers. Long Grove: Wavely Press, Inc.

Chase, Arlen F. y Diane Z. Chase

2006 "Before the Boom: Caracol's Preclassic Era", Research Reports in Belizean Archaeology, 3: 41-57.

2012 Interpreting Ancient Maya Society through Neighborhoods: Investigation of Adjacent Residential Complexes near Caracol's Epicenter: Caracol Archaeological Project Investigations for 2012. Report submitted to the Belize Institute of Archaeology. 
Chase, Diane Z. y Arlen F. Chase

2011 "Ghosts Amid the Ruins: Analyzing Relationships between the Living and the Dead among the Ancient Maya of Caracol, Belize", Living with the Dead: Mortuary Ritual Mesoamerica, pp. 78-101, James L. Fitzsimmons e Izumi Shimada (eds.). Tucson: University of Arizona Press.

Chase, Diane Z.

1988 "Caches and Censerwares: Meaning from Maya Pottery", A Pot For All Reasons: Ceramic Ecology Revisited, pp. 81-104, Louanna Lackey y Charles Kolb (eds.). Philadelphia: Temple University, Laboratory of Anthropology.

Cuevas García, Martha y J. Alvarado Ortega

2012 "El mar de la creación primordial: un escenario mítico y geólogico en Palenque” Arqueología Mexicana, 19 (113): 32-37.

Estrada-Belli, Francisco

2006 "Lightning Sky, Rain, and the Maize God: the Ideology of Preclassic Maya Rulers at Cival, Petén, Guatemala”, Ancient Mesoamerica, 17 (1): 57-78. DOI: $10.1017 /$ S0956536106060068

Fogelin, Lars

2007 "The Archaeology of Religious Ritual”, Annual Review of Anthropology, 36: 5571. DOI:10.1146/annurev.anthro.36.081406.094425

Freidel, David A. y Linda Schele

1988 "Kingship in the Late Preclassic Maya Lowlands: the Instruments and Places of Ritual Power", American Anthropologist, 90 (3): 547-567. DOI: 10.1525/ aa.1988.90.3.02a00020

García Moll, Roberto

1985 Palenque 1926-1945. México: Instituto Nacional de Antropología e Historia.

Goldberg, Paul, Christopher E. Miller, Solveig Schiegl, Bertry Ligouis, Francesco Berna, Nicholas J. Conard y Lyn Wadley

2009 "Bedding, Hearths, and Site Maintenance in the Middle Stone Age of Sibudu Cave, KwaZulu-Natal, South Africa", Archaeological and Anthropological Sciences 1 (2): 95-122.

Hanks, William F.

2013 "Counterparts: Co-presence and Ritual Intersubjectivity", Language \& Communication, 33: 263-277. DOI: 10.1016/j.langcom.2013.07.001.

Harrison-Buck, Eleanor

2004 "Nourishing the Animus of Lived Space through Ritual Caching", K'axob: Ritual, Work, and Family in an Ancient Maya Village, pp. 65-86, Patricia McAnany (ed.). Los Angeles: Cotsen Institute of Archaeology, University of California. 
Houston, Stephen, Claudia Brittenham, Cassyra Mesick, Alexyre Tokovinine y Christina Warinner

2009 Veiled Brightness: A History of Ancient Maya Color. Austin: University of Texas Press.

Humphrey, Caroline y James Alexander Laidlaw

1994 The Archetypal Actions of Ritual: An Essay on Ritual as Action Illustrated by the Jain Rite of Worship. Oxford: Oxford University Press.

Hutson, Scott R. y Richard E. Terry

2006 "Recovering Social and Cultural Dynamics from Plaster Floors: Chemical Analyses at Ancient Chunchucmil, Yucatan, Mexico", Journal of Archaeological Science, 33: 391-404. DOI: 10.1016/j.jas.2005.08.004

Josserand, J. Kathryn

1991 "The Narrative Structure of Hieroglyphic Texts at Palenque", Sixth Palenque Round Table, 1986, pp. 12-31, Merle Greene Robertson (ed.). Norman: University of Oklahoma Press.

Joyce, Rosemary A.

1992 "Ideology in Action: Classic Maya Ritual Practice", Ancient Images, Ancient Thought: The Archaeology of Ideology Proceedings of the Twenty Third Chacmool Annual Conference, pp. 497-505, A. Sean Goldsmith, Sandra Garvie, David Selin y Jeannete Smith (eds.). Calgary, Alberta: University of Calgary.

2008 "Practice in and as Deposition", Memory Work: Archaeologies of Material Practices, pp. 25-39, Barbara J. Mills y William H. Walker (eds). Santa Fe: School for Advanced Research Advanced Seminar Series.

2011 "What Should an Archaeology of Religion Look Like to a Blind Archaeologist?" Archaeological Papers of the American Anthropological Association, 21 (1): 180-188.

Joyce, Rosemary A. y Susan D. Gillespie

2015 "Making Things out of Objects that Move", Things in Motion: Object Itineraries in Anthropological Practice, pp. 3-20, Rosemary A. Joyce and Susan D. Gillespie (eds.). Santa Fe: School for Advanced Research Advanced Seminar Series, Santa Fe Press.

Joyce, Rosemary A. y Joshua Pollard

2010 "Archaeological Assemblages and Practices of Deposition", The Oxford Handbook of Material Culture, pp. 291-309, Dan Hicks y Mary Beaudry (eds.). Oxford: Oxford University Press.

Kidder, Alfred V., Jesse D. Jennings y Edwin M. Shook

1946 Excavations at Kaminaljuyu, Guatemala. Pennsylvania: Pennsylvania State University Press.

Lentz, David L., Jason Yaeger, Cynthia Robin y Wendy Ashmore

2005 "Pine, Prestige, and Politics of the Late Classic Maya at Xunantunich, Belize", Antiquity, 79: 573-585. DOI: 10.1017/S0003598X00114516 
Liendo Stuardo, Rodrigo

2013 Informe Proyecto Regional Palenque (PREP): Temporada 2013. Informe entregado al Instituto Nacional de Antropología e Historia, Mexico.

López Bravo, Roberto

1995 "El Grupo B, Palenque, Chiapas: una unidad habitacional maya del Clásico Tardío”, tesis de licenciatura en Arqueología. México: Escuela Nacional de Antropología e Historia.

2004 "State and Domestic Cult in Palenque Censer Stands", Courtly Art of the Ancient Maya, pp. 256-258. New York: Thames and Hudson.

Maudslay, Alfred P.

1889-1902 Archaeology, Biologia Centrali-Americana. F. Ducane Godman and Osbert Salvin (eds.). London: RH Porter.

Matthews, W., C. A. I. French, T. Lawrence, D. F. Cutler y M. K. Jones

1997 "Microstratigraphic Traces of Site Formation Processes and Human Activities", World Archaeology 29 (2): 281-308. DOI: 10.1080/00438243.1997.9980378

Maxwell, David

1996 "An Analysis of Caches from Four Sites in the Maya Lowlands", tesis para obtener el grado de doctor en Filosofía. Arizona: University of Arizona, Department of Anthropology.

Morehart, Christopher T., David L. Lentz y M. Keith Prufer

2005 "Wood of the Gods: The Ritual Use of Pine (Pinus spp.)", Latin American Antiquity, 16 (3): 255-274. DOI: 10.2307/30042493

Pollard, Joshua

2010 "The Aesthetics of Depositional Practice", World Archaeology 33 (2): 315-333. DOI: $10.1080 / 00438240120079316$

Rands, Robert y Barbara C. Rands

1961 "Excavations in a Cemetery at Palenque", Estudios de Cultura Maya, 1: 87-106. DOI: 10.19130/iifl.ecm.1961.1.199

Richards, Colin y Julian Thomas

1984 "Ritual Activity and Structured Deposition in Later Neolithic Wessex", Neolithic Studies, pp. 189-218, R. Bradley y J. Gardiner (eds.). Oxford: British Archaeological Reports.

Ruz Lhuillier, Alberto

1952 "Exploraciones en Palenque: 1952”, Anales del Instituto de Antropología e Historia, 5 (33): 47-66.

1954 "Exploraciones arqueológicas en Palenque: 1954”, Anales del Instituto Nacional de Antropología e Historia, 6 (10): 117-184. 
1958 "Exploraciones arqueológicas en Palenque: 1958”, Anales del Instituto Nacional de Antropología e Historia, 6 (14): 91-112.

San Román Martín, Elena

2005 "El Clásico Temprano en Palenque a través de su cerámica”, Lakamha’: Boletín Informativo del Museo de Sitio y Zona Arqueológica de Palenque, año 4, segunda época, número 16: 3-8.

Schele, Linda

1986 "The Demotion of Chac-Zutz': Lineage Compounds and Subsidiary Lords at Palenque", Sixth Palenque Round Table, pp. 6-11, Merle Greene Robertson (ed.). Norman: University of Oklahoma Press.

Stone, Andrea J.

2002 "Spirals, Ropes and Feathers", Ancient Mesoamerica, 13: 21-39.

DOI: $10.1017 /$ S0956536102131026

Stuart, David

1998 "The Fire Enters his House": Architecture and Ritual in Classic Maya Texts", Function and Meaning in Classic Maya Architecture, pp. 373-425, Stephen D. Houston (ed.). Washington, D.C.: Dumbarton Oaks.

Swenson, Edward

2015 "The Archaeology of Ritual”, Annual Review of Anthropology, 44: 329-345. DOI: 10.1146/annurev-anthro-102214-013838.

Taschek, Jennifer T. y Joseph W. Ball

1999 "Las Ruinas de Arenal: Preliminary Report on a Subregional Major Center in the Western Belize Valley (1991-1992 excavations)", Ancient Mesoamerica, 10 (2): 215-235.

Taube, Karl A.

2005 "The Symbolism of Jade in Classic Maya Religion”, Ancient Mesoamerica, 16: 23-50. DOI: $10.1017 /$ S0956536105050017

Tozzer, Alfred M.

1941 Landa's Relación de las Cosas de Yucatan, a Translation. Cambridge: Peabody Museum of American Archaeology and Ethnology, Harvard University.

Tringham, Ruth

2000 "The Continuous House: a View from the Deep Past", Beyond Kinship: Social and Material Reproduction in House Societies, pp. 115-134, Rosemary A. Joyce y Susan D. Gillespie (eds.). Philadelphia: University of Pennsylvania Press.

Van Keuren, Scott y Christopher I. Roos

2013 "Geoarchaeological Evidence for Ritual Closure of a Kiva at Fourmile Ruin, Arizona”, Journal of Archaeological Science, 40: 615-625. DOI: 10.1016/j. jas.2012.08.011 
Vogt, Evon Z.

1976 Tortillas for the Gods: A Symbolic Analysis of Zinacanteco Rituals. Salt Lake City: University of Oklahoma Press.

Zidar, Charles y Wayne Elisens

2009 "Sacred Giants: Depiction of Bombacoideae on Maya Ceramics in Mexico, Guatemala, and Belize”, Economic Botany, 63 (2): 119-129. 